\title{
Coherence effects in propagation through photonic crystals
}

\section{A. Mandatori}

\section{Bertolotti \\ C. Sibilia}

\section{B.J. Hoenders}

\section{Scalora}

\author{
Dipartimento di Energetica, University of Roma "La Sapienza", Via A. Scarpa 16, 00161 Roma \\ Dipartimento di Energetica, University of Roma "La Sapienza”, Via A. Scarpa 16, 00161 Roma \\ Dipartimento di Energetica, University of Roma “La Sapienza”, Via A. Scarpa 16, 00161 Roma
}

University of Groningen, Centre for Theoretical Physics and Materials Science Centre, Nijenborgh 4, 9747 AG Groningen, The Netherlands

U.S. Army Aviation and Missile Command, Weapon Sciences Directorate, AMSMI-RD-WS-ST Redstone Arsenal, Huntsville, Alabama 35898-5000

We have analytically studied how a partially coherent quasi plane wave is affected by a photonic crystal structure including a grating. The analysis is presented for spatial and temporal cases showing the possibility to determine the coherence characteristics of the pulse. [D0I: $10.2971 /$ jeos.2006.06005]

Keywords: coherence, photonic crystals, propagation

\section{Introduction}

Random fluctuations of an electromagnetic field are the object of the theory of coherence [1].

In the analysis of imaging systems, the spatial-coherence state of the light source plays an important role; in fact, the degree of spatial coherence of the illuminating source strongly affects the image quality, and numerous criteria, such as spatialfrequency cut-off, two-point resolution, speckle phenomenon, and the diffraction effect at a screen edge, can be used to compare image properties [2]. The ability to asses the state of coherence of an illuminating beam is therefore of great importance.

Perhaps, the earliest works on the theory of partial coherence for the propagation in periodic structures have been the study of the effect of multireflections occurring in optical cavities by E. Wolf [3] and W. Streifer [4].

More recently some attempts have been made to construct a theory of partial coherence for wave propagation in periodic media such as photonic crystals [5]. In the present paper we wish to analyze a 2D-structure which shows a transmission that may be strongly influenced by the spatial coherence properties of a monochromatic beams, so providing a simple tool to give a rough measure of the coherence properties of the beam.
2 COHERENCE THROUGHA TWODIMENTIONAL GRATING SYST E M

We wish to analyze the coherence properties of a $2 \mathrm{D}$ structure including a diffractive element such as a grating. The response of such geometry is strongly influenced by the wavelength of the radiation and therefore it is convenient to speak in spectral terms adopting the concept of spectral visibility and spectral correlations developed by Mandel and Wolf already in 1976 $[6,7]$. The spectral visibility $\vartheta(r, \omega)$ at a given point $P(r)$ is defined as

$$
\vartheta(r, \omega)=\frac{W(r, \omega)_{\max }-W(r, \omega)_{\min }}{W(r, \omega)_{\max }+W(r, \omega)_{\min }}
$$

where $W(r, \omega)$ is the spectral density at the frequency $v$. For a white source of continuous spectral density in a sufficient small interval of frequency $\delta v$, one may assume

$$
W(r, \omega)=W_{\omega}=\cos t
$$

and

$$
I_{\omega}=\int_{\omega}^{\omega+\delta \omega} W\left(\omega^{\prime}\right) d \omega^{\prime}=W_{\omega} \delta \omega
$$

The spectral visibility is then

$$
\vartheta_{\omega}(r)=\frac{I_{\omega}(r)_{\max }-I_{\omega}(r)_{\min }}{I_{\omega}(r)_{\max }+I_{\omega}(r)_{\min }}
$$

To start with, let us consider the most intriguing part of our studied structure made by a grating with a period $d$ along 
the $x$-direction on which a plane monochromatic wave propagating along $z$ and polarized linearly with the electric field vibrating along $y$ (see Figure 1) is impinging. A number of plane waves comes out from the grating (in the figure three waves are shown) with different propagation directions. The outcoming field can be written as

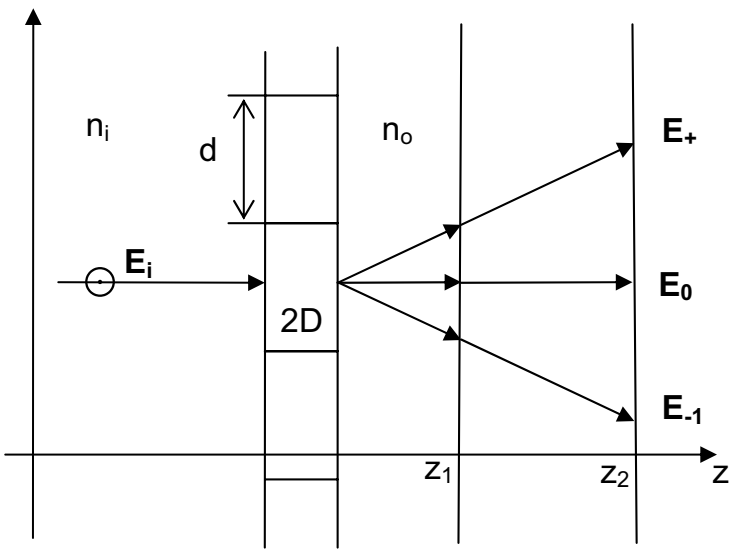

FIG. 1 Representation of a simple 2D grating system with one input plane wave in and three plane waves in output.

$$
E_{\omega}(x, z)=\sum_{n=-\infty}^{\infty} T_{n} e^{-i\left(k_{n x} x+k_{n z} z\right)}
$$

where $T_{n}$ are complex coefficients determined by the boundary conditions of the problem

$$
\begin{aligned}
& k_{n z}=\sqrt{n_{0}^{2} k_{0}^{2}-k_{n x}^{2}} \\
& k_{n x}=n \frac{2 \pi}{d}+n_{i} k_{0} \sin \varphi_{i}=n k_{d}+k_{x 0}
\end{aligned}
$$

$n_{0}$ is the external output medium refractive index, $k_{0}$ is the wave-number in vacuum, $n_{i}$ is the input refractive index, $n$ is an integer number $n=0, \pm 1, \pm 2, \ldots$ and $\varphi_{i}$ is the incoming angle. If the input field is incident normally, the incoming angle $\varphi_{i}$ is zero and to have homogeneous plane waves in transmission one must have

$$
n_{0}^{2} k_{0}^{2}-k_{n x}^{2} \geq 0
$$

Therefore the $n t h$ order diffracted wave will propagate in the output semispace only if

$$
n \leq n_{0} \frac{d}{\lambda}
$$

If the output medium has a refractive index $n_{0}$ and only $2 n+1$ orders are wanted, then

$$
n \leq n_{0} \frac{d}{\lambda}<n+1
$$

In the observation plane at some distance $z_{0}$ from the $2 \mathrm{D}$ system, the output field is made by the sum of a finite number of plane waves which interfere with each other. The calculation of the total field in the case of fully coherent waves is quite easy. To consider more complex cases let us start from the spatial frequency integral representation of the output field so that the output field $E_{u}(x)$ can be written as the inverse Fourier transform on the plane $z$

$$
\begin{aligned}
& E_{\text {out }}(x)=\int \sum_{n=-N}^{N} T_{n}\left(k_{x}\right) \times \\
& e^{-i\left[\left(n k_{d}+k_{x}\right) x+\sqrt{n_{0}^{2} k_{0}^{2}-\left(n k_{d}+k_{x}\right)^{2}} z\right]} E_{i}\left(k_{x}\right) d k_{x}
\end{aligned}
$$

or in a more compact way

$$
E_{\text {out }}(x)=\int T\left(k_{x}, x\right) E_{i}\left(k_{x}\right) e^{-i k_{x} x} d k_{x}
$$

where

$$
T\left(k_{x}, x\right)=\sum_{n=-N}^{N} T_{n}\left(k_{x}\right) e^{-i\left[n k_{d} x+\sqrt{n_{0}^{2} k_{0}^{2}-\left(n k_{d}+k_{x}\right)^{2}} z\right]} .
$$

We are now in the position to obtain the mutual coherence function for the field at frequency $v$

$$
\begin{gathered}
\Gamma_{\text {out }}^{(\omega)}\left(x_{1}, x_{2}\right)=<E_{\text {out }}\left(x_{1}\right) E_{\text {out }}\left(x_{2}\right)^{*}>= \\
=<\int T\left(k_{x 1}, x_{1}\right) E_{i}\left(k_{x 1}\right) e^{-i k_{x 1} x_{1}} d k_{x 1} \times \\
\quad \int T\left(k_{x 2}, x_{2}\right)^{*} E_{i}\left(k_{x 2}\right)^{*} e^{i k_{x 2} x_{2}} d k_{x 2}>
\end{gathered}
$$

which can be written as

$$
\begin{aligned}
& \Gamma_{\text {out }}^{(\omega)}\left(x_{1}, x_{2}\right)=\iint \Gamma_{i}^{(\omega)}\left(k_{x 1}, k_{x 2}\right) \times \\
& T\left(k_{x 1}, x_{1}\right) T\left(k_{x 2}, x_{2}\right)^{*} e^{-i k_{x 1} x_{1}} e^{i k_{x 2} x_{2}} d k_{x 1} d k_{x 2}
\end{aligned}
$$

where

$$
\Gamma_{i}^{(\omega)}\left(k_{x 1}, k_{x 2}\right)=<E_{i}\left(k_{x 1}\right) E_{i}^{*}\left(k_{x 2}\right)>
$$

is the spatial cross-spectral function of the incident field of pulsation $\omega$. If the two points $x_{1}$ and $x_{2}$ coincide, $x_{1}=x_{2}=x$, then $\Gamma_{\text {out }}^{(\omega)}(x, x)$ is the output intensity and we have

$$
\begin{gathered}
I_{\omega}(x)=\iint \Gamma_{i}^{(\omega)}\left(k_{x 1}, k_{x 2}\right) T\left(k_{x 1}, x\right) T\left(k_{x 2}, x\right)^{*} \times \\
e^{-i\left(k_{x 1}-k_{x 2}\right) x} d k_{x 1} d k_{x 2} .
\end{gathered}
$$

If we assume that the input plane wave is a stationary process that depends on the two spatial variables only through their difference $x^{\prime \prime}-x^{\prime}=\delta, \Gamma$ is a function of the difference between the points $x^{\prime}$ and $x^{\prime \prime}$ only and we may write for

$$
\begin{gathered}
<E_{i}\left(k_{x 1}\right) E_{i}\left(k_{x 2}\right)^{*}>=\iint<E_{i}\left(x^{\prime}\right) E_{i}\left(x^{\prime \prime}\right)^{*}>\times \\
e^{i k_{x 1} x^{\prime}} e^{-i k_{x 2} x^{\prime \prime}} d x^{\prime} d x^{\prime \prime}
\end{gathered}
$$

the expression

$$
<E_{i}\left(k_{x 1}\right) E_{i}\left(k_{x 2}\right)^{*}>=\Gamma_{i}^{(\omega)}\left(k_{x 1}\right) \delta\left(k_{x 1}-k_{x 2}\right)
$$

and substituting Eq(18) in Eqs.(15) and (16) we obtain

$$
I_{\omega}(x)=\int \Gamma_{i}^{(\omega)}\left(k_{x 1}\right)\left|T\left(k_{x 1}, x\right)\right|^{2} d k_{x 1} .
$$

The calculation of Eq.(19) requires the knowledge of $T_{n}\left(k_{x}\right)$. The simplest way to operate is by numerical integration. To improve the speed of calculation of the integral, a database has been created with a number of values of $T_{n}\left(k_{x}\right)$ for different $k_{x}$ values and given polarization (TE or TM). From the database by interpolation all the points of $T_{n}\left(k_{x}\right)$ are derived 
and are used to perform the integral Eq.(19) for any position $z_{0}$ and any correlation function $\Gamma_{i}$.

A simple example may be considered by assuming to have an input wave with a Gaussian correlation function

$$
\Gamma_{i}^{(\omega)}(x)=e^{-x^{2} / 2 \sigma^{2}} e^{-i k_{x 0} x}
$$

where $k_{x 0}$ has been defined in Eq.(6). The Fourier transform can be written as

$$
\Gamma_{i}^{(\omega)}\left(k_{x}\right)=A \sigma e^{-\frac{1}{2} \sigma^{2}\left(k_{x}-k_{x 0}\right)^{2}}
$$

being $\sigma$ (with the dimension of a length), a measure of the spatial coherence of the wave. For values of $\sigma>>\lambda$, being $\lambda$ the input wavelength $\Gamma_{i}\left(k_{x}\right)$ is well approximated by a Dirac $\delta$ function and Eq.(19) can be written as

$$
I(x)=\Gamma_{i}^{(\omega)}\left(k_{x 0}\right)\left|T\left(k_{x 0}, x\right)\right|^{2} .
$$

Except for a multiplicative constant, Eq.(22) is exactly the intensity distribution on the plane $z$ ( $z$ is implicit in the function $T$ ) of a fully spatially coherent field. We have important effects due to partial coherence only if the function $\Gamma_{i}\left(k_{x}\right)$ is wide enough not to be approximated by a Dirac $\delta$ function. In this case, we have a combined effect due to the partial coherence of the incoming field and the geometrical properties of the system. If $\sigma>>\lambda$, the geometrical parameters of the 2D structure are not so important. Consider however Eq.(19) and write down the general form of $T$ as in Eq.(12)

$$
T\left(k_{x}, x\right)=\sum_{n=-N}^{N} T_{n}\left(k_{x}\right) e^{-i\left[n k_{d} x+\sqrt{n_{0}^{2} k_{0}^{2}-\left(n k_{d}+k_{x}\right)^{2}} z\right]} .
$$

We have to consider that in the sum Eq.(23) the terms $T_{n}\left(k_{x}\right)$ are constant with respect to the spatial coordinates $x$ and $z$. They are produced only by the angular spectral properties of the $2 \mathrm{D}$ structure. The exponential term is the propagator for the single plane waves that from the $2 \mathrm{D}$ system propagate till the $z$ plane, and it may produce changes in $T\left(k_{x}, x\right)$ as the observation plane distance $z$ is changed.

In Figure 1 (where for simplicity only three waves are considered of the $2 N+1$ ones in output) we consider two $z$ planes where to calculate the intensity by Eq.(19). The propagator for the $n$-plane wave in output is written as $K_{n}=$ $e^{-i\left[n k_{d} x+\sqrt{n_{0}^{2} k_{0}^{2}-\left(n k_{d}+k_{x}\right)^{2}} z\right]}$ $k_{x}$ values the number of turns of the vector $K_{n}$ on the complex plane (and therefore the number of the phase cycles) is greater as the $z$ plane is moved far apart. This means that Eq.(23), that gives the form of the interference fringes on the $z$ plane in the case of a completely coherent field Eq.(19), is more sensible to $k_{x}$ as the $z$ plane moves farthest away. In particular, the fringes tend to disappear by increasing $z$. In Figure 1 the interference fringes will be more visible on the plane $z_{1}$ than on $z_{2}$. The calculation shows that the visibility of fringes depends on the ratio $z / \sigma$. For $z<3 \sigma$ the fringes are still visible, for $z>3 \sigma$ the visibility tends to zero. This is a characteristic linked to the mathematical and physical properties of the propagator $K_{n}$, more than to the geometrical characteristics of the $2 \mathrm{D}$ system.

\section{ANALYSIS OF THE SPATIAL AND TEMPORAL COHERENCE ONA GRATING}

Taking into account Figure 1, we proceed to analyze the general case of a space and time partially coherent input plane wave on a $2 \mathrm{D}$ system made by a grating with period $d$. We begin from the integral representation in terms of spatial and temporal frequencies of the output field on the generic $z$ plane.

$$
\begin{gathered}
\left.E_{\text {out }}(x, t)=\iint \sum_{n=-N}^{N} T_{n}\left(k_{x}, \omega\right) e^{-i\left[n k_{d} x+\sqrt{n_{0}^{2} k_{0}^{2}-\left(n k_{d}+k_{x}\right)^{2}} z\right.}\right]_{\times} \\
E_{i}\left(k_{x}, \omega\right) e^{-i k_{x} x} e^{i \omega t} d k_{x} d \omega
\end{gathered}
$$

or using Eq.(12)

$$
E_{\text {out }}(x, t)=\iint T\left(k_{x}, \omega, x\right) E_{i}\left(k_{x}, \omega\right) e^{-i k_{x} x} e^{i \omega t} d k_{x} d \omega .
$$

We now proceed to derive the mutual coherence function

$$
\begin{aligned}
& \Gamma_{\text {out }}\left(x_{1}, x_{2} ; t_{1}, t_{2}\right)=<E_{\text {out }}\left(x_{1}, t_{1}\right) E_{\text {out }}\left(x_{2}, t_{2}\right)^{*}>= \\
& \iint T\left(k_{x_{1}}, \omega_{1}, x_{1}\right) E_{i}\left(k_{x_{1}}, \omega_{1}\right) e^{-i k_{x_{1}} x_{1}} e^{i \omega_{1} t_{1}} d k_{x_{1}} d \omega_{1} \times \\
& \iint T\left(k_{x_{2}}, \omega_{2}, x_{2}\right)^{*} E_{i}\left(k_{x_{2}}, \omega_{2}\right)^{*} e^{i k_{x_{2}} x_{2}} e^{-i \omega_{2} t_{2}} d k_{x_{2}} d \omega_{2}
\end{aligned}
$$

that can be written as

$$
\begin{aligned}
& <E_{\text {out }}\left(x_{1}, t_{1}\right) E_{\text {out }}\left(x_{2}, t_{2}\right)^{*}>= \\
& \iiint \int<E_{i}\left(k_{x_{1}}, \omega_{1}\right) E_{i}\left(k_{x_{2}}, \omega_{2}\right)^{*}>\times \\
& T\left(k_{x_{1}}, \omega_{1}, x_{1}\right) T\left(k_{x_{2}}, \omega_{2}, x_{2}\right)^{*} \times \\
& e^{-i k_{x_{1}} x_{1}} e^{i k_{x_{2}} x_{2}} e^{i \omega_{1} t_{1}} e^{-i \omega_{2} t_{2}} d k_{x_{1}} d k_{x_{2}} d \omega_{1} d \omega_{2}
\end{aligned}
$$

or in a more compact way

$$
\begin{aligned}
& \Gamma_{\text {out }}\left(x_{1}, x_{2}, t_{1}, t_{2}\right)= \\
& \quad \iiint \int \Gamma_{i}\left(k_{x_{1}}, k_{x_{2}}, \omega_{1}, \omega_{2}\right) T\left(k_{x_{1}}, \omega_{1}, x_{1}\right) T\left(k_{x_{2}}, \omega_{2}, x_{2}\right)^{*} \times \\
& e^{-i k_{x_{1}} x_{1}} e^{i k_{x_{2}} x_{2}} e^{i \omega_{1} t_{1}} e^{-i \omega_{2} t_{2}} d k_{x_{1}} d k_{x_{2}} d \omega_{1} d \omega_{2} .
\end{aligned}
$$

To derive the spectral correlation function $\Gamma_{i}$ in spatial and temporal frequencies we can proceed as for Eqs.(17), (18) obtaining

$$
\begin{gathered}
\Gamma_{i}\left(k_{x_{1}}, k_{x_{2}}, \omega_{1}, \omega_{2}\right)=<E_{i}\left(k_{x_{1}}, \omega_{1}\right) E_{i}\left(k_{x_{2}}, \omega_{2}\right)^{*}>= \\
\Gamma_{i}\left(k_{x_{1}}, \omega_{1}\right) \delta\left(k_{x_{1}}-k_{x 2}\right) \delta\left(\omega_{1}-\omega_{2}\right) .
\end{gathered}
$$

Replacing Eq.(29) inside Eq.(28) we have

$$
\begin{gathered}
\Gamma_{\text {out }}\left(x_{1}, x_{2}, t_{1}, t_{2}\right)=\iint \Gamma_{i}\left(k_{x_{1}}, \omega_{1}\right) T\left(k_{x_{1}}, \omega_{1}, x_{1}\right) \times \\
T\left(k_{x_{1}}, \omega_{1}, x_{2}\right)^{*} e^{-i k_{x_{1}}\left(x_{1}-x_{2}\right)} e^{i \omega_{1}\left(t_{1}-t_{2}\right)} d k_{x_{1}} d \omega_{1} .
\end{gathered}
$$

That in the case of fully spatial coherent field in input becomes

$$
\begin{gathered}
\Gamma_{\text {out }}\left(x_{1}, x_{2}, t_{1}, t_{2}\right)=\int \Gamma_{i}\left(\omega_{1}\right) T\left(\omega_{1}, x_{1}\right) \times \\
T\left(\omega_{1}, x_{2}\right)^{*} e^{i \omega_{1}\left(t_{1}-t_{2}\right)} d \omega_{1}
\end{gathered}
$$

while for a fully time coherent field we have

$$
\begin{aligned}
& \Gamma_{\text {out }}\left(x_{1}, x_{2}, t_{1}, t_{2}\right)=\Gamma_{\text {out }}\left(x_{1}, x_{2}\right)= \\
& \quad \int \Gamma_{i}\left(k_{x_{1}}\right) T\left(k_{x_{1}}, x_{1}\right) T\left(k_{x_{1}}, x_{2}\right)^{*} e^{-i k_{x_{1}}\left(x_{1}-x_{2}\right)} d k_{x_{1}} .
\end{aligned}
$$


In the case of a plane wave with central pulsation $\omega_{0}$, stationary Gaussian coherent function in space and stationary Lorentzian coherent function in time, the correlation function is written as (we define $x=x_{1}-x_{2}, \tau=t_{1}-t_{2}$ )

$$
\Gamma_{i}(x, \tau)=e^{-\frac{x^{2}}{2 \sigma_{x}^{2}}} e^{-\frac{|\tau|}{\sigma_{t}}} e^{-i k_{x_{0}} x} e^{i \omega_{0} \tau}
$$

where $\sigma_{x}$ and $\sigma_{t}$ are related to the space coherence and linewidth of the pulse. The Fourier transform in space and in time (except for a constant factor) is

$$
\Gamma_{i}\left(k_{x_{1}}, \omega_{1}\right)=\frac{2 e^{-\frac{1}{2} \sigma_{x}^{2}\left(k_{x_{1}}-k_{x_{0}}\right)^{2}}}{\sigma_{t}\left[\left(\omega-\omega_{0}\right)^{2}+\frac{1}{\sigma_{t}^{2}}\right]} .
$$

Considering Eq.(30), the output intensity for a partial coherent input field is

$$
I(x)=\iint \Gamma_{i}\left(k_{x_{1}}, \omega_{1}\right)\left|T\left(k_{x_{1}}, \omega_{1}, x\right)\right|^{2} d k_{x_{1}} d \omega_{1}
$$

with $\Gamma_{i}$ given by Eq.(34).

\section{AN EXAMPLE; A PHOTONIC CRYSTAL WITH A GRATING AS A DEFECT}

We now consider a more complex 2D structure made by alternating a number of layers of different refractive index, with a simple grating at the centre.

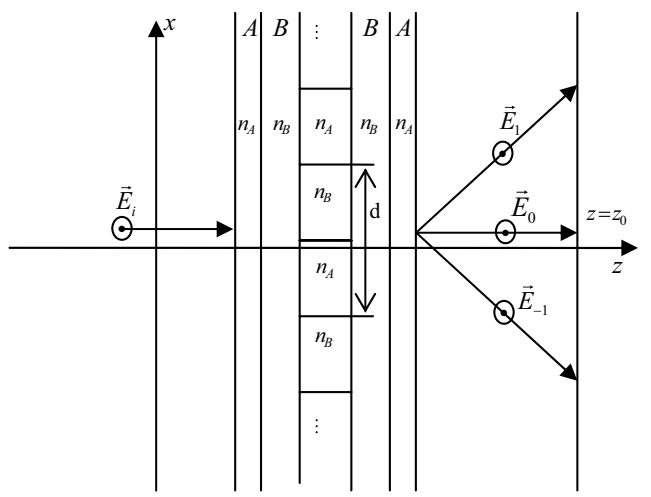

FIG. 2 Representation of a grating between two multilayers at $\lambda_{0} / 4$.

The general structure is shown in Figure 2. The structure may be represented in a symbolic way as $A B(2 D) B A$ with obvious meanings of the symbols. The simulations that are reported below have used for the different parameters the following values: $n_{A}=2.5, n_{B}=1.5$; all the thicknesses of the 1 D-layers are chosen to have optical path $\lambda_{0} / 4$ where $\lambda_{0}=0.6 \mu \mathrm{m}$. The grating represents a defect in the middle of the 1D-layers and its thickness is $a_{g}=0.25 \mu \mathrm{m}$. The period $d$ has been chosen $d=a \lambda_{0}$ with $a=1.8$; with this choice of $d$ and $\lambda_{0}$ it is clear that only three plane waves are present in the output, as it is schematized in Figure 2. The period $d$ includes two regions of refractive index $n_{A}$ and $n_{B}$ of equal extension.

Figure 3 shows the transmission spectrum T: in Figures 3(a), 3(b) and 3(c) are shown for normal incidence the transmission spectra in (a) for the diffracted zero order only, in (b) for the -1 and +1 orders (in case of normal incidence these two spectra are equal), in (c) the average power that can be measured on a generic output plane (obviously the average power is equal to $\left.\left|T_{-1}\right|^{2}+\left|T_{0}\right|^{2}+\left|T_{+1}\right|^{2}\right)$.
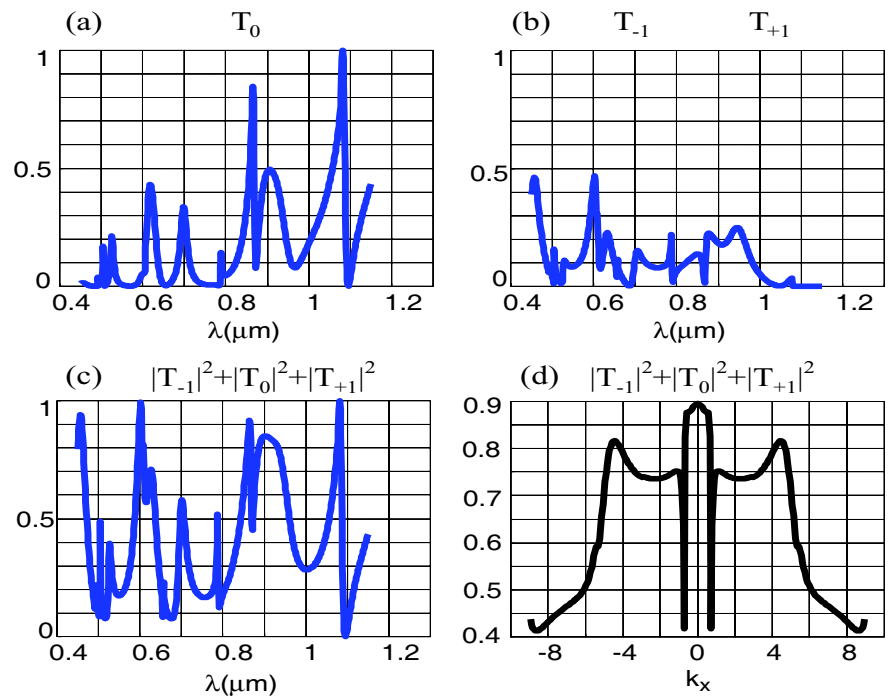

(d) $\left|\mathrm{T}_{-1}\right|^{2}+\left|\mathrm{T}_{0}\right|^{2}+\left|\mathrm{T}_{+1}\right|^{2}$

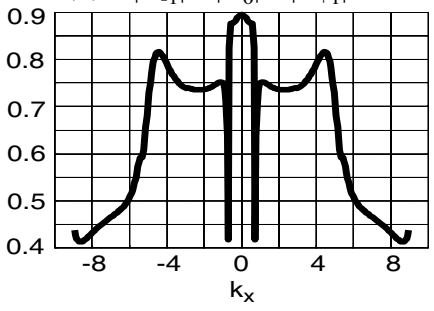

FIG. 3 Transmissions for the system $A B(2 D) B A$ of Figure 2. (a) transmission spectrum of zero order for normal incidence; (b) transmission spectrum of -1 and +1 orders for normal incidence (for normal incidence they are the same); (c) average transmission power at the output plane for normal incidence; (d) average transmission power at the output plane for different angles of incidence $\left(k_{x}=k_{x}\left(\phi_{i}\right)\right.$, where $k_{x}$ is the $x$-component of the wave vector $\left.k_{0}\right)$, in this last case the wavelength is fixed at $\lambda=0.6 \mu \mathrm{m}$

In Figure 3(d) the transmission is given as a function of the wave vector $k_{x}$ for $\lambda=0.6 \mu \mathrm{m}$. For the sake of simplicity, we will limit our considerations and calculations to the case of a monochromatic wave in the pass band of the structure.

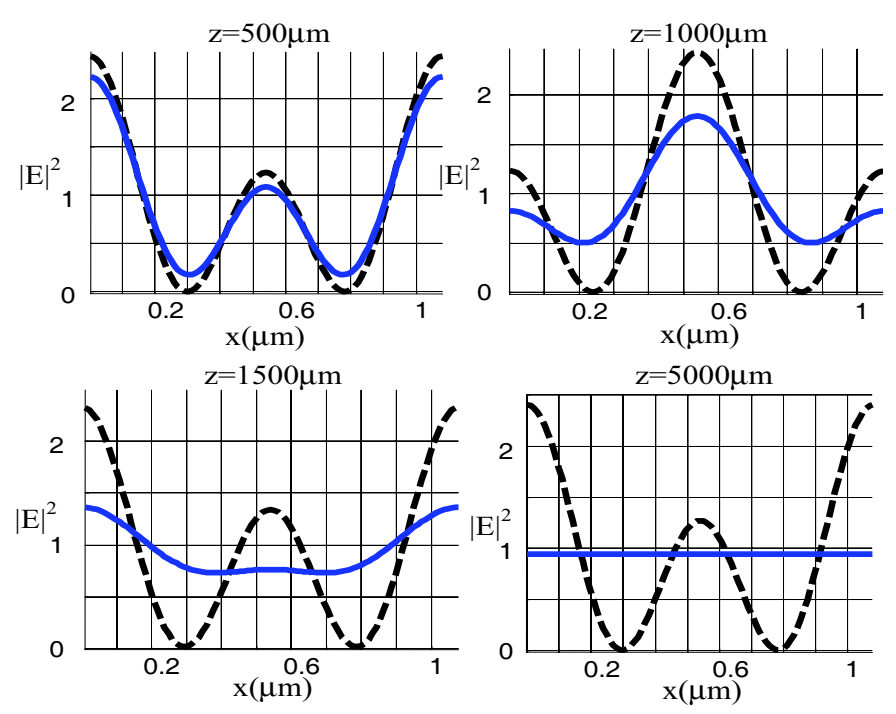

FIG. 4 Square modulus of transmitted electric field at several $z$ planes, the dashed curves refer to a completely coherent input plane wave, the solid lines refer to a partially coherent input plane wave, with $\sigma=1000 \mu \mathrm{m}$. 
Figures 4 show the interference fringes obtained on a plane at four different distances $z$. In each figure two curves are shown: the dashed one shows the case of a fully coherent field $(\sigma=\infty)$, the solid one shows the fringes for a partially coherent field with $\sigma=1000 \mu m$.

Simple inspection shows that by increasing the distance $z$ the fringe contrast for the partially coherent field decreases becoming eventually zero at great distance, while the full coherent field gives fringes at any distance.
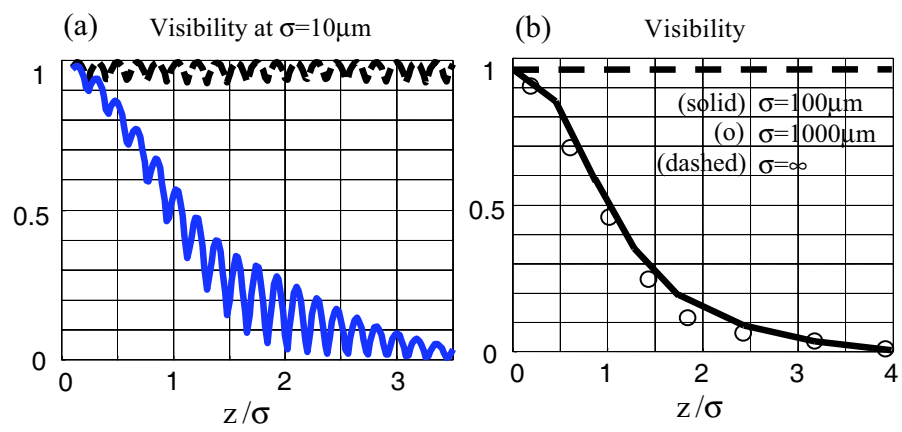

FIG. 5 Visibility curves for different values of $\sigma$ as a function of $z$. (a) The dashed-curve refers to a completely coherent input plane wave, the solid-curve refers to a partially input plane wave with $\sigma=10 \mu \mathrm{m}$. (b) Visibility average for different values of $\sigma$. Solid line $\sigma=100 \mu \mathrm{m}$, (o) $\sigma=1000 \mu \mathrm{m}$, dashed line $\sigma=\infty$. The system is $A B(2 D) B A$.

Visibility curves following the definition of Eq.(4) are shown in Figures 5 for the three different cases $\sigma=10,100$ or $1000 \mu \mathrm{m}$. For comparison the visibility for a fully coherent field is also shown. As we can see, for all cases the visibility of the fringes due to a partial coherent plane wave (solid line) decreases to zero after some $\sigma$ steps, while for a complete coherent plane wave the visibility (dashed line) keeps a high value for the entire range. Figure 5(a) shows that the visibility has small fluctuations as a function of $z / \sigma$. These fluctuations have been smeared out in Figure 5(b), because they are very fast when $\sigma$ increases.
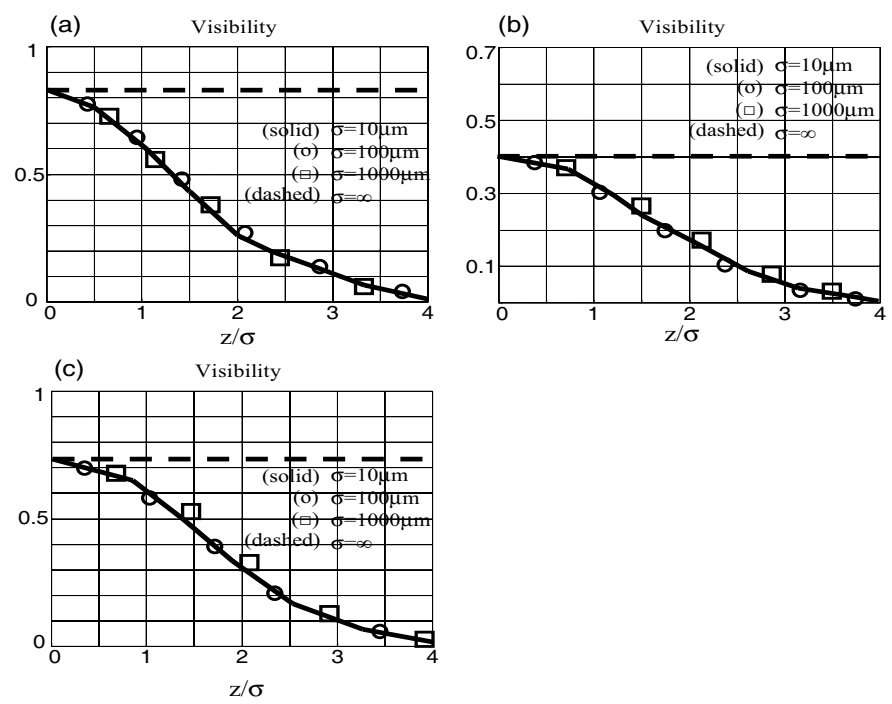

FIG. 6 Visibility curves for different values of $\sigma$ as a function of $z$. The dashed line refers to a completely coherent input plane wave, the solid line refers to a partially input plane wave with $\sigma=10 \mu \mathrm{m}$, (o) is for $\sigma=100 \mu \mathrm{m}$, ( $\square$ ) is for $\sigma=1000 \mu \mathrm{m}$. (a) The system is $A B A B(2 D) B A B A$; (b) the system is $A B A B A B(2 D) B A B A B A$; (c) the system is $A B A B A B A B(2 D) B A B A B A B A$;

We now can see what happens if we change some parameters in the structure of Figure 2. The first case is obtained by the addition of some pairs of 1D-layers, obtaining structures more selective on wavelength. We represent these systems with symbolic notation as: (a) $A B A B(2 D) B A B A$, (b) $A B A B A B(2 D) B A B A B A$, (c) $A B A B A B A B(2 D) B A B A B A B A$. For all the three cases the physical dimensions are the same of Figure 2. The visibility curves for the cases (a), (b), (c) are shown in Figures 6(a), 6(b), 6(c) respectively, they are obtained for three values of $\sigma(\sigma=10 \mu m, \sigma=100 \mu m, \sigma=1000 \mu m)$. The visibility for a fully coherent wave is also shown on the three figures as a dashed line, and it remains constant as a function of $z / \sigma$.

A second situation we will treat is the one in which we change the thickness of the grating keeping all others physical parameters of the system as shown in Figure 2. The number of 1D-layers is four at each of the two sides of the grating. With symbolic notation the system is $A B A B(2 D) B A B A$. We choose two cases where the thickness is: (a) $a_{g}=0.05 \mu \mathrm{m}$ and (b) $a_{g}=0.45 \mu \mathrm{m}$. These cases have to be compared with the first one $\left(a_{g}=0.25 \mu \mathrm{m}\right)$ treated in Figure 6(a). The visibility curves for the cases (a) and (b) are shown in Figures 7(a), 7(b) respectively. Here too, they are obtained for three values of $\sigma$ $(\sigma=10 \mu m, \sigma=100 \mu m, \sigma=1000 \mu m)$, and visibility for a fully coherent field is shown (dashed line).

A third very interesting situation arises if the period $\mathrm{d}$ of the grating is changed. We have seen that, if $\mathrm{d}$ is larger than $\lambda$, more orders of diffraction are present. It means that more beams interfere in the final output plane and we may expect to have very complex fringes. This is indeed what happens. We have simulated a geometry as in Figure 2 with $\lambda_{0}=0.4 \mu \mathrm{m}$ and $d=a \lambda_{0}$ with $a=12.5 \mu \mathrm{m}$. The thicknesses of the layers are as usual $\lambda_{0} / 4$, and the grating has an arbitrary thickness of $0.25 \mu \mathrm{m}$. The other parameters are unchanged.
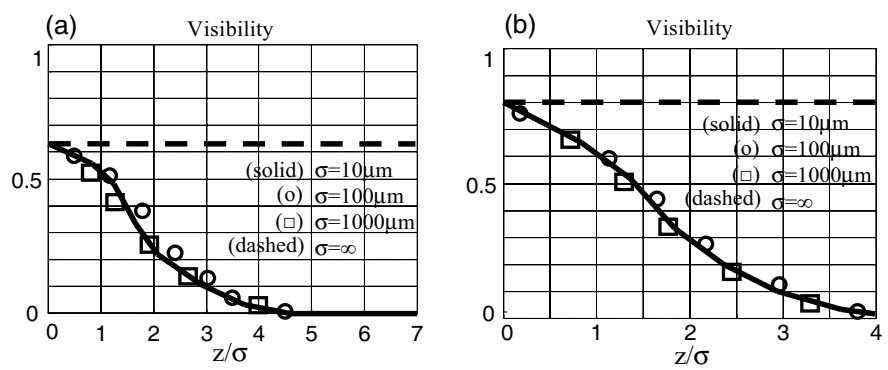

FIG. 7 Visibility curves for different values of $\sigma$ as a function of $z$. The dashed line refers to a completely coherent input plane wave, the solid line refers to a partially input plane wave with $\sigma=10 \mu \mathrm{m}$, (o) is for $\sigma=100 \mu \mathrm{m}$, ( $\square$ ) is for $\sigma=1000 \mu \mathrm{m}$. (a) The system is $A B A B(2 D) B A B A$, with the thickness of the $2 \mathrm{D}$ defect $a=0.005 \mu \mathrm{m}$; (b) the system is $A B A B(2 D) B A B A$, with $a_{g}=0.45 \mu \mathrm{m}$. 
(a)
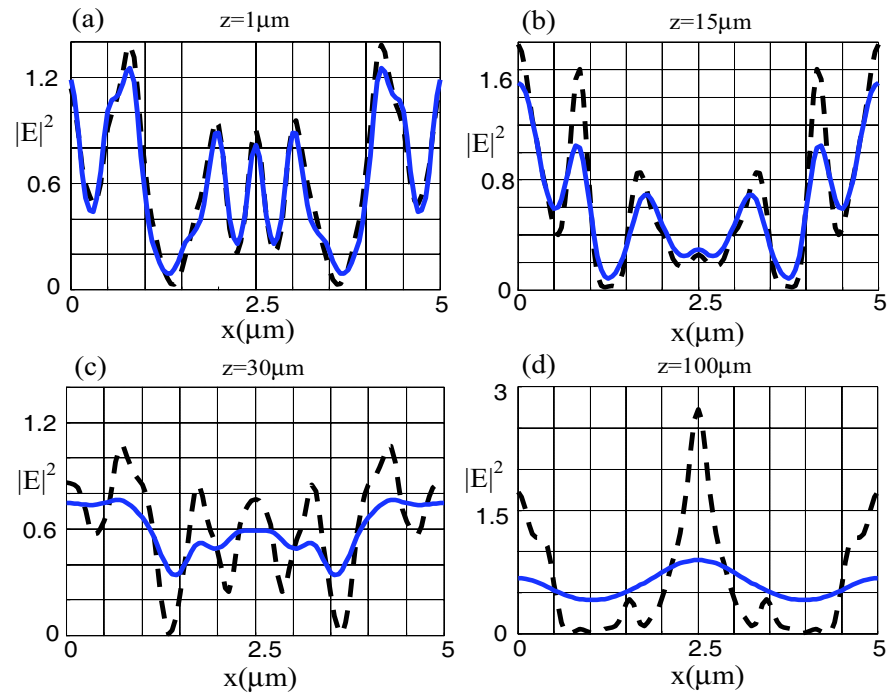

FIC. 8 Fringes as a function of normalized distance $z / \sigma$ for $\mathrm{d}=12.5 \times 1.4 \mu \mathrm{m}$ and $\sigma=$ $10 \mu \mathrm{m}$. The dashed curves refer to a fully coherent beam.

The obtained fringes are shown in Figures 8 assuming a coherence dimension of the incoming beam $\sigma=10 \mu \mathrm{m}$. Again in each graph the fringes produced by a fully coherent beam are also drawn. As expected the fringes have a rather complex distribution, but also in this case at some distance $\mathrm{z}$ the fringes of the partially coherent source disappear (Figure 8(d)). It may be observed that, by increasing the period d of the grating, the distance at which fringes disappear is still linked to $\sigma$, but it is not a few times $\sigma$, but larger. The exact functional dependence of $\mathrm{z}$ on $\sigma$ has however not been determined, and should be explored in a next paper.

A final example is shown in Figures 9 which have been obtained for the geometry of Figure 2 with $d=2.0 \mu \mathrm{m}, \lambda_{0}=$ $0.532 \mu \mathrm{m}, n_{A}=\mathrm{TiO}_{2}, n_{B}=\mathrm{SiO} 2$, and $\sigma=1 \mathrm{~mm}$. Also in this case the general behavior is confirmed with the validity of the comments already done to Figures 8 . The visibility is shown in Figure 10 where the fluctuations have not been smeared out.
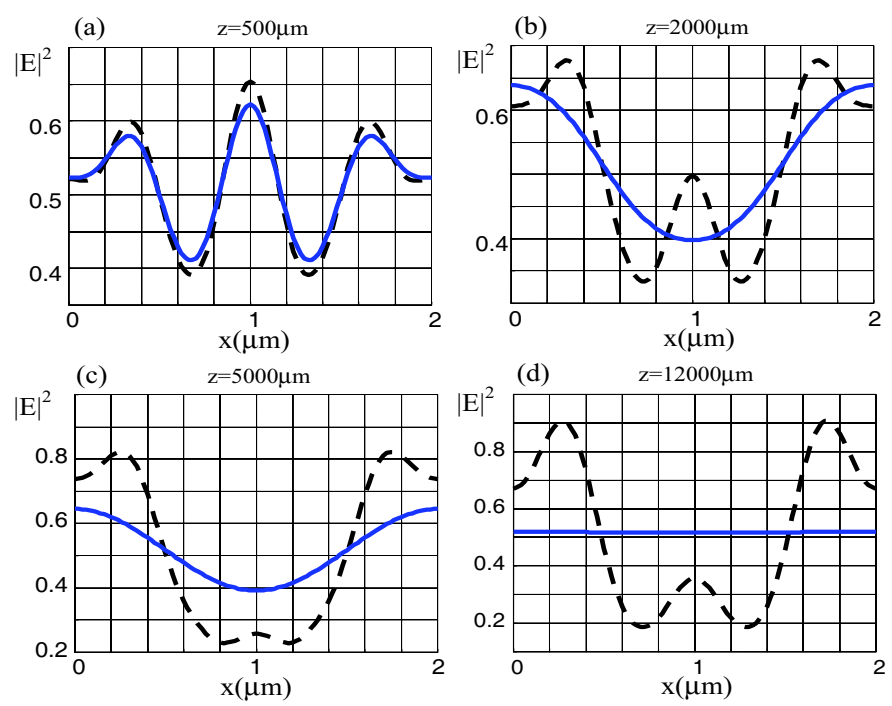

FIG. 9 Fringes as a function of normalized distance $z / \sigma$ for $d=2.0 \mu \mathrm{m}, \sigma=0.532 \mu \mathrm{m}$. The dashed curves refer to a fully coherent beam.

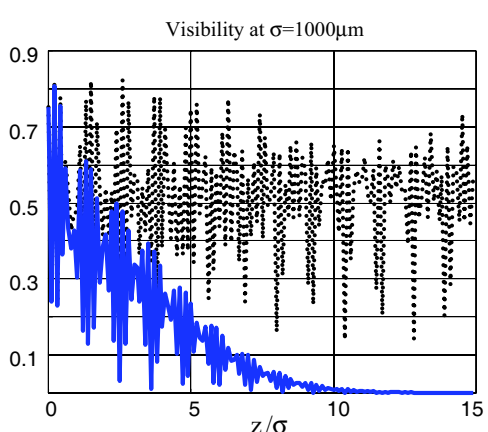

FIG. 10 Visibility for the case of Figure 9. $\sigma=1 \mathrm{~mm}$, as a function of normalized distance $z / \sigma$. The dashed curve refers to the case of a fully coherent beam.

\section{C O N CLUSIONS}

A photonic crystal with a grating as a defect may be strongly sensitive to the coherence of the incoming beam. The simulations that have been done show that the position of the intensity maxima changes as a function of $z$ as it may be expected and the fringe visibility is always small because the three interfering plane waves have not optimized amplitude. Therefore even in the fully coherent case the distribution of intensity outside the structure exhibits fringes of low visibility that changes as a function of $z$ in a no oscillating way. The mean fringe visibility of the partially coherent source is however always lower than for the fully coherent source and eventually goes to zero for values of $z>>\sigma$. Figures 6 and 7 show that, in the case of partially coherent fields, the behaviour of visibility depends on the ratio $z / \sigma$ rather than simply on $z$. This means that for larger coherence dimensions of the incoming beam, fringes are observed in the image after the filter for proportionally higher distances.

This behavior could find application for a very simple and rapid inspection of the spatial coherence and temporal coherence properties of a source. Observing the intensity distribution on a plane moving far away from the structure, at some distance of the order of some times $\sigma$, fringes should disappear.

This method is stable and fast to find the coherence degree of a beam because we need only to put the filter in front of the beam and detect the output field at several distance. The distance at which the fringes disappear is a rough measure of $\sigma$.

\section{References}

[1] M. Born and E. Wolf, Principles of Optics (Pergamon, New York, 1970).

[2] J. W. Goodman, Introduction a l'optique de Fourier (Masson, Paris, 1972).

[3] E. Wolf, Phys. Lett. 3, 166 (1963).

[4] W. Streifer, J. Opt. Soc. Am. 56, 1481 (1966).

[5] B.J. Hoenders and M. Bertolotti, Theory of partial coherence for weakly periodical media,

[6] L. Mandel, E. Wolf, J. Opt. Soc. Am. 66, 529 (1976).

[7] E. Wolf, Opt. Lett. 8, 250 (1983). 